\title{
La fiction à substrat professionnel (FASP) : un(e) média(tion) pas comme les autres
}

\section{Rosalyn de Charentenay}

\section{(2) OpenEdition}

Édition électronique

URL : http://journals.openedition.org/asp/1962

DOI : 10.4000/asp. 1962

ISBN : 978-2-8218-0384-8

ISSN : 2108-6354

\section{Éditeur}

Groupe d'étude et de recherche en anglais de spécialité

Édition imprimée

Date de publication : 1 octobre 2001

Pagination : 203-213

ISSN : 1246-8185

\section{Référence électronique}

Rosalyn de Charentenay, « La fiction à substrat professionnel (FASP) : un(e) média(tion) pas comme les autres », ASp [En ligne], 31-33 | 2001, mis en ligne le 20 novembre 2010, consulté le 19 avril 2019. URL : http://journals.openedition.org/asp/1962 ; DOI : 10.4000/asp.1962

Ce document a été généré automatiquement le 19 avril 2019

Tous droits réservés 


\title{
La fiction à substrat professionnel (FASP) : un(e) média(tion) pas comme les autres
}

\author{
Rosalyn de Charentenay
}

\section{Introduction}

Une étude récente (Petit 2000) a présenté le postulat de l'émergence d'un nouveau genre, baptisé fiction à substrat professionnel ou FASP. La FASP, qui trouve ses racines dans les genres du thriller ou du roman policier, se distingue de ces derniers par la présence permanente et essentielle d'un réel professionnel qui en fait le véritable substrat de l'œuvre de fiction. L'importance de la FASP pour les enseignants de langue de spécialité réside dans la pertinence que ce genre peut avoir pour les enseignements élaborés dans le domaine de la langue pour spécialistes d'autres disciplines (LANSAD). Pourrait-on ainsi trouver dans la FASP un moyen de réconcilier la littérature et l'enseignement de la langue spécialisée? Quelles sont les caractéristiques significatives de la FASP qui la rendraient pertinente et quelles sont les applications pratiques? Ces questions sont abordées dans une étude que nous avons conduite sur l'emploi d'une œuvre de fiction dans l'enseignement/apprentissage de la terminologie et de la culture juridique américaine. Dans cet article, nous exposons les réflexions induites par cette étude en examinant dans un premier temps le rôle de la fiction dans l'enseignement/apprentissage de la langue spécialisée, puis en présentant le travail fait sur l'élaboration d'un cours de langue destiné aux étudiants en maîtrise de droit à l'IUP ${ }^{1}$ Juriste d'Entreprise à partir du roman de John Grisham, The Rainmaker². 


\section{Le rôle de la fiction dans l'enseignement/ apprentissage de la langue spécialisée}

2 Si le titre de cette communication suggère qu'il existe des différences remarquables entre le roman dans son emploi en tant que support pédagogique et certains autres supports, ce n'est pas un hasard. Les « supports de diffusion massive de l'information », presse, radio, télévision, cinéma, qui correspondent aux média selon la définition du Dictionnaire Encyclopédique Larousse, fournissent depuis longtemps des sujets d'étude dans le domaine LANSAD. A l'inverse, le livre ${ }^{3}$, qui est aussi inclus dans cette liste des médias, a été quasiment exclu de l'élaboration des cours de langue spécialisée et, par conséquent, de la recherche dans ce domaine, depuis la naissance du secteur LANSAD dans les années 1980. Dans cette partie, nous examinons les raisons de cette exclusion et réfléchissons à la question suivante : la médiation offerte par la FASP, pourrait-elle rouvrir la voie à une réconciliation entre la littérature et l'enseignement/ apprentissage de la langue de spécialité ?

\subsection{La littérature et la langue de spécialité}

3 Dans les années 1980, on constate l'absence presque totale de l'enseignement de la littérature dans les programmes d'anglais de spécialité. La littérature, ainsi que la grammaire et la traduction, sont supplantées par d'autres méthodes considérées comme plus adaptées aux exigences de l'époque. On estime que l'étude de la grammaire et de la traduction s'inspire plutôt de la linguistique et que la littérature dépend du domaine de la critique littéraire. La période où une certaine élite apprenait la langue par le biais de la littérature paraît révolue. La justification principale de cette exclusion repose sur l'idée que la littérature n'est qu'un frein dans les processus d'apprentissage (Widdowson 1985 :180). D'abord le manque d'intérêt que les apprenants non-spécialistes de langue montrent pour l'étude de la littérature constitue un obstacle majeur car une méthode d'apprentissage qui ne sollicite pas l'intérêt de l'étudiant a peu de chances de réussir (Widdowson 1983: 91). De surcroit, la littérature classique a souvent une écriture complexe et obscure qui entraîne une baisse de motivation de l'apprenant face aux difficultés rencontrées, perçues comme inutiles et ennuyeuses. Par ailleurs, le langage littéraire présente souvent des formes d'usage peu communes, obligeant alors l'apprenant à fournir dans la phase de réception un travail de compréhension qu'il doit ensuite rejeter comme modèle pour sa propre production. Dans une telle situation la légitimité de l'enseignement peut être mise en question dans l'esprit de l'apprenant.

La tendance qui nourrit alors la croissance du domaine de LANSAD va de pair avec celle qui voit diminuer l'importance de la littérature. Les besoins pratiques de l'apprenant sont devenus la préoccupation principale des enseignants en langue de spécialité. La nécessité d'élaborer des enseignements liés directement au domaine d'application de l'apprenant oblige la recherche à examiner dans un premier temps les registres, les genres et les tâches liés au domaine d'application. Les méthodes et méthodologies proposées préconisent l'emploi du texte en omettant la littérature (Widdowson 1985 : 181). Il n'empêche que certains auteurs du domaine LANSAD vantent toujours l'importance de la littérature et appellent à un retour de son emploi en langue de spécialité. Tout d'abord, la responsabilité, qui est celle de l'enseignant, d'éduquer (educate) ses étudiants et de les 
ouvrir au monde est mise en avant. Les objectifs pédagogiques ne doivent pas se limiter à la simple formation dans les compétences requises pour leur avenir professionnel (training). La littérature aurait un effet d'humanisation qui fait partie de l'éducation.

Desirable effects of this broad, humanistic interpretation of education, to which the study of literature contributes, include: the discovery that English can be a beautiful language (Spack 1988), "growth in emotional awareness and maturity » (Hirvela \& Boyle 1988 : 181), development of « empathy » (Robson 1989 : 27), habits of enquiry and speculation, critical reasoning and the conscious testing of inferences or hypotheses (Gajdusek 1988: 233). The claim is explicitly made that these latter skills are transferable : « Such training helps [students] in other courses which demand logical reasoning, independent thinking, and careful analysis of text » (Spack $1985: 721)$. (Horowitz $1990: 162)^{4}$

En réponse à cet argument, certains auteurs, en particulier Horowitz, ne sont pas convaincus de la pertinence en ce qui concerne les enseignements en langue de spécialité. Horowitz insiste sur le fait qu'il est primordial que les étudiants reçoivent une éducation qui soit enrichissante humainement, mais il se demande si le cours de langue est le lieu approprié pour ce genre d'enseignement. Il reconnaît que le débat sur l'emploi de la littérature en langue de spécialité est en réalité un microcosme du débat centré sur les communautés de discours (discourse communities) et pose la question de la possibilité de suivre une autre voie pour enrichir les capacités des étudiants au niveau du développement de la logique et du raisonnement. Pour cela, il propose qu'une équipe d'enseignants et d'apprenants soit formée afin que chacun devienne ethnographe dans la culture concernant le domaine de spécialité. La préconisation sous-jacente à cette proposition est que les enseignants, qui sont majoritairement formés en littérature, oublient l'enseignement qu'ils ont reçu. En effet, les enseignants doivent s'informer sur la discipline d'application de leurs étudiants afin d'être à même d'employer les outils auxquels les étudiants sont habitués pour élaborer des cours pertinents et efficaces.

Les propos de Horowitz sont intéressants dans la mesure où il rappelle que l'élément essentiel lié à la décision d'utiliser la littérature en tant que support pédagogique de la langue est l'étudiant : les facteurs à prendre en compte sont ses besoins, ses préférences, son niveau d'étude, la constitution du groupe. En somme, la conclusion à en tirer serait que la littérature classique a des avantages indéniables, mais présente des inconvénients liés à son peu de pertinence pour le domaine d'application. Cela nous laisse supposer que s'il existait un genre littéraire jetant un pont entre les deux rives (les besoins d'une expérience éducative générale offerte par la littérature et les besoins de la communauté de spécialité) les arguments contre son emploi disparaîtraient.

\subsection{La fiction à substrat professionnel (FASP)}

7 Il est en effet possible que la notion de FASP apporte une solution aux hésitations exprimées par Horowitz par rapport à l'emploi de la littérature en langue de spécialité. Petit (2000) démontre qu'il existe un genre auquel il donne l'appellation de fiction à substrat professionnel ou FASP. Ce genre regroupe plusieurs variétés de fictions commerciales telles que le thriller médical, le thriller juridique et le thriller militaire. L'ensemble de ces œuvres se distingue du genre, thriller spécialisé, "par la présence permanente et nécessaire, à la fois dans le contenu narratif et dans l'écriture elle-même, de la représentation prégnante d'un réel professionnel qui en fait le véritable substrat de la fiction » (2000: 58). L'auteur signale que le manque d'attention critique à ce genre par 
rapport à d'autres genres est dû principalement à son essor récent. Par conséquent, il suggère que la nature de la FASP en fait un objet légitime et pertinent pour l'anglistique de spécialité (2000: 57$)$. Il met en lumière l'existence d'éléments de caractérisation externe et d'éléments de caractérisation interne de la FASP, qui indiquent l'appartenance de certains auteurs et de leurs œuvres à ce nouveau genre. Une analyse approfondie de ce postulat dépasse le cadre de cet article. Toutefois nous tenons à signaler son impact sur la réorientation du débat concernant l'exploitation de la littérature ${ }^{5}$ dans les enseignements de langue de spécialité.

8 Dans le cas présent, le livre The Rainmaker, de John Grisham, a fait l'objet d'une étude menée auprès des étudiants en droit, niveau maîtrise, de l'IUP Juriste d'Entreprise. Il ne s'agissait pas, bien évidemment, de prouver l'existence d'un nouveau genre en n'étudiant qu'une seule œuvre de fiction. Toutefois, ce tâtonnement a permis de mettre en lumière des éléments de réflexion pour des recherches ultérieures. De plus, cette étude a permis d'analyser dans quelle mesure ce texte de fiction, qui fait partie d'un genre habituellement extérieur au domaine des discours spécifiques, pourrait constituer un support pédagogique légitime et pertinent dans une situation d'enseignement / apprentissage en langue de spécialité dans le domaine juridique.

\section{Objet de la recherche : la didactisation d'une œuvre de fiction}

9 L'étude menée sur la didactisation de The Rainmaker s'est faite sous forme de rechercheaction. L'hypothèse centrale qui a guidé notre recherche était la suivante : les étudiants en maitrise à l'IUP Juriste d'Entreprise-Juriste International peuvent, à partir d'une œuvre de fiction, assimiler les mécanismes et les concepts de la procédure civile américaine tout en intégrant les aspects culturels associés. L'inspiration pour ce projet de recherche-action trouve ses racines dans la création du premier IUP destiné à former les étudiants juristes d'entreprise. Avec la création d'une nouvelle formation, de nouveaux besoins sont à identifier et à prendre en compte. Les besoins spécifiques d'un groupe d'étudiants appellent souvent à l'innovation par les enseignants de langue de spécialité dans l'élaboration des cours (Haramboure 1996). Étant donné que les innovations doivent être raisonnées et fondées sur des principes scientifiques, le projet de recherche-action avait comme objectif l'observation méthodique d'une démarche pédagogique sur la didactisation d'un ouvrage de fiction.

10 Comme tout enseignement dans le domaine de LANSAD, le point de départ de la recherche consistait à déterminer les besoins des apprenants, ce qui impliquait de prendre en considération le niveau de langue des étudiants, leur maturité, leurs objectifs professionnels, et la constitution du groupe. Nous avons retenu le modèle de Chancerel et Richterich (1980) car l'aspect tripartite de l'analyse des besoins semblait être le plus approprié. Une enquête par questionnaire a été effectuée auprès des étudiants, et des entretiens approfondis ont été conduits avec des représentants de l'institution (responsables de la formation) et des représentants de la profession (juristes d'entreprise). Les éléments concluants qui ont été dégagés de ces enquêtes pour l'élaboration du cours sont les suivants : il existait un consensus entre les trois acteurs concernant l'importance pour les étudiants d'acquérir un niveau d'anglais correspondant aux exigences de leur profession ; un cours fondé sur une matière du droit américain était 
conforme aux souhaits de l'institution; la procédure civile américaine est une matière pertinente pour les futurs juristes d'entreprise.

11 Une fois les besoins spécifiques définis, il faut choisir les supports pédagogiques. La collecte, la sélection et la production de documents sont des préoccupations principales de l'enseignant LANSAD. Par ailleurs, le choix des documents à utiliser est fait par rapport aux objectifs fixés par l'enseignant (Mémet 1995). La difficulté rencontrée dans le cas présent a été de trouver un ou plusieurs supports pédagogiques permettant une transmission de savoir aux étudiants, tout en leur donnant la possibilité d'élargir leurs compétences linguistiques ainsi que leurs compétences culturelles, ce qui est à la fois intéressant et motivant pour les apprenants. Après avoir étudié et analysé les différentes possibilités qui se présentaient (films, manuels de droit, documents professionnels, textes de loi, articles de presse, cas de jurisprudence, œuvres de fiction), nous avons décidé de concentrer l'étude sur l'emploi d'une œuvre de fiction. Les qualités déterminantes étaient liées à la pertinence, à l'originalité, à l'intérêt et à la motivation que ce support pouvait susciter chez les étudiants.

\subsection{La langue de spécialité juridique et The Rainmaker}

Dans cette partie, nous analyserons les caractéristiques du roman The Rainmaker qui le rendent légitime et pertinent en tant qu'outil pédagogique dans un enseignement en langue de spécialité. L'œuvre de fiction étant le seul support pédagogique choisi, on doit s'assurer que ce livre contient une description assez précise des règles de procédure civile ainsi que des indicateurs de la culture professionnelle pour que les étudiants puissent en retirer l'essentiel. Tout d'abord, pour vérifier le premier point, la présence suffisante des règles de procédure civile, nous avons comparé le contenu de l'ouvrage de fiction avec celui d'un manuel de droit (Burnham 1995). Le schéma donné en annexe montre les trois phases principales de la procédure civile : avant le procès, durant le procès, après le procès (pre-trial, trial et post-trial). La comparaison révèle la présence d'une description de toutes les étapes principales de phase 1 (pre-trial) et de phase 2 (trial). Parmi les techniques pour débouter un procès (voir annexe, colonne 2), seule la motion to dismiss a ététraitée.

13 Une fois qu'on a établi la présence de la terminologie juridique nécessaire pour comprendre les différentes étapes de la procédure civile, la mise en situation professionnelle est l'élément clef qui distingue le roman du manuel de droit. Le vocabulaire utilisé est souvent moins technique dans le roman, et la définition des termes est parfois incomplète ou absente, mais l'aspect concret du fonctionnement du système est bien illustré. Parmi les techniques employées par l'auteur pour communiquer un réel professionnel on trouve des définitions dans le texte, des explications du raisonnement juridique derrière une règle de procédure, des illustrations de la stratégie professionnelle dans le choix d'action, et des explications des principes du droit américain. Ces différentes techniques sont illustrées ci-dessous par des citations du livre de Grisham.

\subsubsection{Définition de termes juridiques dans le texte}

14 Les depositions, interrogatories et requests for admission sont les trois outils principaux employés dans la phase Discovery de la partie avant-procès (pre-trial) d'une action en justice aux Etats-Unis. Leur présence dans le livre est particulièrement importante pour 
les étudiants en droit français car la façon d'échanger les renseignements se déroule différemment en France.

Depositions

Kipler asks Donny Ray if he's ready to proceed, then instructs the court reporter to swear him. He promises to tell the truth. Since he's my witness, and this is for evidentiary purposes as opposed to the normal unbridled fishing expedition, my direct examination of him must conform to the rules of evidence. (236)

Interrogatories

With these files, I prepared a lengthy list of interrogatories, written questions one party sends another to be answered in writing and under oath. (230-231)

Requests for admissions

A set of requests for admissions. The latter is a neat device to force an opposing party to admit or deny certain facts set forth in writing within thirty days. If the facts are not defined, then they are forever deemed admitted. (243-244)

\subsubsection{Explication du raisonnement derrière une règle de procédure}

Fast-tracking

In response to years of criticism by laymen and lawyers alike, the rules of civil procedure were changed not long ago in an effort to speed up justice. Sanctions for frivolous lawsuits were increased. Mandatory deadlines for pre-trial maneuvering were imposed. Judges were given more authority to ramrod litigation, and they were also encouraged to become more active in settlement negotiations. Lots or rules and laws were implemented, all in an effort to streamline the civil justice system.

Created in this mass of new regulations was a procedure commonly known as "fast-tracking ", designed to bring certain cases to trial faster than others. The term "fast-tracking» was instantly added to our legal jargon. The parties involved can request that their case be fast-tracked, but this seldom happens. It's a rare defendant who'll agree to a speedy trip to the courtroom. So the judge has the authority to do it on his own volition. It's usually done when the issues are clear, the facts are sharply defined but hotly in dispute, and all that's needed is a jury's verdict. (213-214)

\subsubsection{Illustration de la stratégie professionnelle}

Strategically, it's to their advantage to postpone the deposition until Donny Ray dies, thus preventing the jury from seeing his withered face and hearing his tortured voice. And there's little doubt that, between the five of them, they guys can orchestrate enough excuses to stall until I die of old age. (226)

\subsubsection{Explication de principes de droit}

The day after Kipler was sworn in, Drummond filed a motion to remove the case to the federal court. He's claiming that Bobby Ott, the agent who sold the policy to the Blacks, has been included as a defendant for all the wrong reasons. Ott, we think, is still a resident of Tennessee. He's a defendant. The Blacks, residents of Tennessee, are the plaintiffs. Complete diversity of citizenship between the parties must exist for federal jurisdiction to apply. 
Ott defeats diversity because, as we allege, he lives here, and for this reason and this reason alone, the case cannot be a federal one. Drummond filed a massive brief in support of his argument that Ott should not be a defendant. (220-221)

\subsection{Observations}

Les outils de recherche employés pour le recueil des données étaient les suivants :

- Pré-test de connaissances sur la procédure civile américaine.

- Post-test de connaissances sur la procédure civile américaine.

- Journal de bord individuel élaboré par l'étudiant.

- Observation des activités par l'enseignant.

- Grille d'observation sur l'acquisition de la terminologie juridique.

L'expérience a montré que les outils les plus utiles pour l'analyse de l'efficacité de cette méthode d'enseignement sont le journal de bord individuel tenu par chaque étudiant, et les grilles d'observation tenues par l'enseignant. Les observations étaient centrées sur l'ouvrage en tant qu'outil pédagogique, et sur la méthodologie pédagogique employée. Nous résumerons ici les opinions des étudiants quant à leur vécu et nous ferons référence au journal de bord que chacun a tenu pendant la durée du cours.

Il faut rappeler que The Rainmaker comporte 434 pages réparties sur 53 chapitres. Une des préoccupations majeures des étudiants était le temps de lecture, de douze heures, pour l'étudiant le plus rapide, jusqu'à trente heures, pour le moins rapide, pour faire une première lecture. Ensuite, pour une compréhension correcte des termes d'anglais juridique ainsi que d'anglais général, il a fallu au moins une relecture, et dans certains cas, trois relectures. La recherche de mots dans le dictionnaire a aussi demandé beaucoup de temps aux étudiants. Néanmoins, certains étudiants ont exprimé leur satisfaction de pouvoir enrichir leur vocabulaire de cette manière. Cette expérience a surtout montré aux étudiants qu'ils sont capables de lire un livre de plus de 400 pages en anglais.

17 L'un des avantages du roman par rapport à un livre de droit est la composante culturelle. Tous les étudiants ont souligné combien il est important de placer la procédure civile dans un contexte culturel. Chaque étudiant a sélectionné les différents points qui ont attiré son attention. Les thèmes abordés incluent le rôle du juge, la vie d'étudiant en faculté de droit, les questions de déontologie des avocats et le rôle de l'avocat.

\section{Conclusion}

18 L'emploi d'une œuvre de fiction comme support pédagogique principal dans l'élaboration d'un cours de langue fondé sur un contenu juridique a été une nouvelle expérience pour les étudiants en maîtrise de droit à l'IUP Juriste d'entreprise. Grâce à cet outil, les étudiants ont pu accéder à la terminologie de la procédure civile américaine ainsi qu'au contexte culturel dans lequel elle se déroule. L'exploitation de ce support est rendue possible par la présence d'un réel professionnel dans l'intrigue et dans l'écriture. Par conséquent, le roman se prête facilement à l'élaboration de tâches authentiques qui permettent l'assimilation des concepts et de la terminologie juridiques.

Le détournement d'un roman à des fins pédagogiques dans un cours de langue demande beaucoup de travail préalable de la part de l'enseignant et des étudiants. L'enseignant doit approfondir le sujet à traiter et procéder au choix du contenu à enseigner. Les 
étudiants, qui n'ont peut-être pas l'habitude d'une lecture extensive en langue anglaise doivent passer un grand nombre d'heures à lire et à relire le texte. Ils feront appel aux stratégies d'apprentissage qu'ils ont acquises tout au long de leurs études pour repérer, organiser et assimiler la matière. Cela signifie qu'un niveau moyen ou avancé en anglais général est nécessaire à l'emploi de cet outil et requiert un certain niveau de maturité de la part des étudiants.

\section{BIBLIOGRAPHIE}

Burnham, William. 1995. Introduction to the Law and Legal System of the United States. St. Paul, Minn: West Publishing Co.

Chancerel, Jean-Louis et René Richterich. 1980. Identifying the Needs of Adults Learning a Foreign Language. Strasbourg: Council of Europe. Reprinted Oxford : Pergamon Press.

Grisham, John. 1995. The Rainmaker. New York :Doubleday.

Haramboure, Françoise. 1996. «L'autonomie des enseignants de langue de spécialité : un gage d'innovation raisonnée ». ASp 11-14, 265-271.

Horowitz, Daniel. 1990. « Fiction and non-fiction in the ESL/EFL classroom: does the difference make a difference ? ». English for Specific Purposes 9/2, 161-168.

Kelly, Renata J. et Lakshamy Krisham. 1995. « 'Fiction talk' in the ESP classroom ». English for Specific Purposes 14/1, 77-86.

Mémet, Monique. 1995. «Content-based instruction in Electrical Engineering: Collecting, organising, and utilising data ». ASp 7-10, 407-420.

Petit, Michel. 1999. « La fiction à substrat professionnel : une autre voie d'accès à l'anglais de spécialité ». ASp 23-26, 1-20.

Widdowson, Henry G. 1983. Learning Purpose and Language Use. Oxford : Oxford University Press.

Widdowson, Henry G. 1985. « Literature teaching: The teaching, learning and study of literature ». InQuirk R. et al. (dir.), English in the World: Teaching and learning the language and literatures. Cambridge : Cambridge University Press, 180-196.

\section{Références bibliographiques complémentaires}

Allwright, Dick. 1993. «Integrating 'research' and 'pedagogy'. Appropriate Criteria and Practical Possibilities ». In Edge, Julian \& Keith Richards (dir.), Teachers Develop Teachers Research, Papers on classroom research and teacher devlopment. Oxford : Heinemann, 125-135.

Allwright, Dick et Kathleen Bailey. 1991. Focus on the Language Classroom: An Introduction to Classroom Research for Language Teachers. Cambridge : Cambridge University Press.

American Corporate Counsel Association. 2000. « A comity of errors, Europeans discover discovery ». ACCA Docket, 33-42.

Bertin, Jean-Claude. 1994. «L'enseignant, le professionnel et l'apprenant-confrontation des cultures et choix des matériaux pédagogiques ». ASp 5-6, 78. 
Brinton, Donna, Marguerite Ann Snow et Marjorie Bingham Wesche. 1989. Content-based second language instruction. New York : Newbury House.

Federal Rules of Civil Procedure - 1994-1995. 1994. St. Paul, Minn. : West Publishing Co.

Flowerdew, John. 1993. « Content-based language instruction in a tertiary setting ». English for Specific Purposes 12/2, 121-138.

Gairns, Ruth et Stuart Redman. 1986. Working With Words: A Guide to Teaching and Learning Vocabulary. Cambridge : Cambridge University Press.

Galonnier, Bernard. 1996. « Les Law Reports de La House of Lords et du Privy Council -1993. L'anglais du droit : terminologie, rhétorique et aspects culturels ». Thèse de doctorat de l'Université Toulouse 1.

Glannon, Joseph. 1994. Civil Procedure. Examples and Explanations, $2^{\mathrm{e}}$ édition. New York : Mathew Bender.

Hutchinson, Tom and Alan Waters. 1987. English for Specific Purposes: A Learning-Centred Approach. Cambridge : Cambridge University Press.

Jacob, Grace P. 1987. «Cultural competence in the ESP curriculum ». English for Specific Purposes $6 / 3,203-218$.

Kasper, Loretta Frances. 1997. « The impact of content-based instructional programs on the academic progress of ESL students ». English for Specific Purposes 16/4, 309-320.

Naturel, Mireille. 1995. Pour la littérature - De l'extrait à l'œuvre. Paris : CLE International.

Sallenave, Danièle. 1997. À quoi sert la littérature? Entretien avec Philippe Petit, Paris : Les éditions Textuel.

Underhill, William. 1999. « Long arm of the lawyers: Global companies want global law firms ». Newsweek June 7, 54. 


\section{ANNEXES}

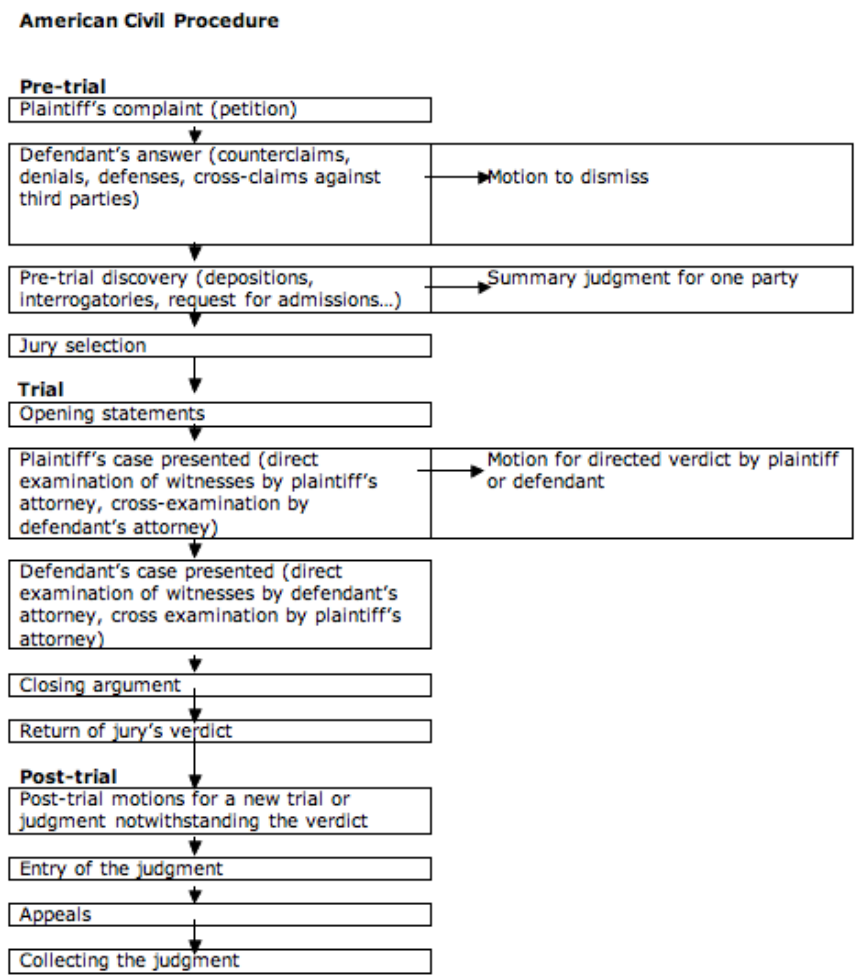

\section{NOTES}

1. Institut Universitaire Professionnalisé

2. Voir aussi Petit (2000), qui examine l'existence de la FASP dans plusieurs domaines (médical, militaire, juridique...) et propose comme exemple de la FASP juridique, ce roman, qui répondrait aux critères de base pour définir ce nouveau genre.

3. Nous faisons particulièrement référence ici aux livres de fiction.

4. Les références dans cette citation sont les suivantes : Gajdusek, L. 1988, «Toward wider use of literature in ESL; why and how », TESOL Quarterly 22, 227-257 ; Hirvela, A. \& J. Boyle, 1988. «Literature courses and student attitudes ", ELT Journal 42, 179-184; Robson, A.E. 1989, "The use of literature in ESL and culture-learning courses in US colleges ", TESOL Newsletter 23.4, 25-27 ; Spack, R. 1985, «Literature, reading, writing, and ESL: bridging the gaps ", TESOL Quarterly 19, 703-725 ; Spack, R. 1988, « The author responds to Braine », TESOL Quarterly 22, 703-705.

5. Il faut préciser que le terme de littérature est employé dans son acception la plus large. Nous n'entrons pas dans le débat qui se pose sur la définition de ce qui constitue la «vraie» littérature. 


\section{RÉSUMÉS}

Cette étude se propose d'examiner divers aspects liés à l'élaboration d'un cours d'anglais juridique intégré dans lequel une œuvre de fiction sert de support principal. Les caractéristiques qui permettent son adaptation en tant que véhicule de savoirs juridiques et culturels sont analysées à la lumière des études sur l'émergence d'un genre à substrat professionnel.

In this study we will examine various aspects related to the implementation of an integrated language and content course in a foreign language setting, wherein a work of legal fiction is used as the primary course book. The characteristics which render the novel appropriate as a source of legal knowledge and culture are analyzed in the light of studies concerning the emergence of a new professionally-based genre.

\section{INDEX}

Mots-clés : anglais de spécialité, anglais juridique, didactique, fiction juridique, rechercheaction, substrat professionnel

Keywords : action research, didactics, ESP, legal English, legal fiction, professional substratum

\section{AUTEUR}

\section{ROSALYN DE CHARENTENAY}

Rosalyn de Charentenay est attachée temporaire d'enseignement et de recherche à l'Université de Toulouse 1. 